Fi ni te el ement anal ysi s of nonl i near dynamic magnet i c fi el d wi th dc component in the har noni c domai $n$

\begin{tabular}{|l|l|}
\hline 著者 & $\begin{array}{l}\text { Yanada Sot oshi , Bi ri nger P.P. , H rano K, } \\
\text { Bessho Kazuo }\end{array}$ \\
\hline $\begin{array}{l}\text { j our nal or } \\
\text { publ i cat i on ti t l e }\end{array}$ & I EEE Tr ansact i ons on Magget i cs \\
\hline vol une & 26 \\
\hline number & 5 \\
\hline page range & $2199-2201$ \\
\hline year & $1990-10-01$ \\
\hline URL & ht t p: //hdl . handl e. net /2297/48301 \\
\hline
\end{tabular}




\title{
FINITE ELEMEN'T ANALYSIS OF NONLINEAR DYNAMIC MAGNETIC FIELD WITH DC COMPONENT IN THE HARMONIC DOMAIN
}

\author{
S. YAMADA, $\uparrow$ P.P. BIRINGER, K. HIRANO and K.BESSHO \\ Faculty of Technology, Kanazawa University, Kodatsuno 2-40-20, Kanazawa, Japan \\ $\uparrow$ Dept. of Electrical Engineering, University of Toronto, Ontario, M5S 1A4, Canada
}

\begin{abstract}
This paper describes the harmonic balance finite element method to analyze steady-state dynamic nonlinear problems with dc magnetization. The feature of the approach is that the fleld calculation is carried out in the harmonic domain. We discuss the analysis of a pot-core type transformer for high frequency converters.
\end{abstract}

\section{INTRODUCTION}

We have proposed the harmonic balance finite element method (HBFEM) for the steady-state analysis of nonlinear dynamic magnetic fields in the harmonic domain(frequency domain)[1]. However, the earlier published IIBFEM can not be used unless the system operates with symmetrical ac waveform. Therefore, it is impossible to analyze transformers for dc-dc converters and smoothing reactors. The reason is that all variables are approximated by the sum of the fundamental component and odd-order harmonics. Here the dc component and even-order harmonics are also considered, thus the HBFEM is extended to more general nonlinear dynamic problems.

The paper describes the formulation of the HBFEM for nonlinear field problems with superposition of dc- and acmagnetizations. We apply this method to the analysis of both a simple smoothing reactor and the pot-core type transformer for high frequency converter.

\section{FORMULATION}

We consider nonlinear eddy-current problems and make the following assumptions:

(1) The field is 2-dimensional.

(2) The problem is quasi-stationary and the displacement current is not considered.

(3) The saturation and hysteresis characteristic of the core are nonlinear.

(4) The material is isotropic.

(5) All variable are in time-periodic steady-state.

Using Galerkin's method, the vector potential $A=(0,0, A)$ in 2-D Cartesian coordinates satisfies the following:

$$
\iint_{n}\left\{\frac{\partial N_{i}}{\partial x}\left(\nu \frac{\partial A}{\partial x}\right)+\frac{\partial N_{i}}{\partial y}\left(\nu \frac{\partial A}{\partial y}\right)\right\} d x d y-\iint_{n}\left(J_{0}+J_{c}\right) N_{i} d x d y=0
$$

where $\nu$ is the magnetic reluctivity. $N_{i}$ is the shape function of the first-order triangular element as a weighting function. The eddy-current density $J_{e}$ is expressed as

$$
J_{e}=-\sigma\left(\frac{\partial A}{\partial t}+\frac{\partial \phi}{\partial z}\right)
$$

where $\phi$ is the scalor potential and $\sigma$ is the conductivity. In the eddy-current region, the following complementary condition is salisfied:

$$
\iint_{\mathbf{n}} J_{\mathbf{e}} d S=0
$$

According to the Weierstrass Approximation Theory, any periodic continuous variable can be approximated by orthonor- mal functions of finite order within arbitrary error. The assumption (5) states that the vector potential $A$ and other variables can be expressed by the trigonometric function. Therefore, vector potential, applied current density, and flux density are expressed as

$$
\begin{gathered}
A^{i}(t)=A_{0}^{i}+\sum_{n=1,2,3 \ldots} A_{n z}^{i} \sin (n \omega t)+A_{n c}^{i} \cos (n \omega t) \\
J_{0}(t)=J_{0}+\sum_{n=1,2,3 \ldots} J_{n t} \sin (n \omega t)+J_{n c} \cos (n \omega t) \\
B_{z}^{e}(t)=B_{x 0}^{t}+\sum_{n=1,2,3 \ldots} B_{z n z}^{t} \sin (n \omega t)+B_{z n c}^{t} \cos (n \omega t) \\
B_{y}^{t}(t)=B_{y 0}^{t}+\sum_{n=1,2,3 \ldots} B_{y, n}^{i} \sin (n \omega t)+B_{y n c}^{t} \cos (n \omega t)
\end{gathered}
$$

According to the assumption (3), the magnetizing curve of it core is expressed as

$$
H(B)=H_{s a l}(B)+H_{h y s}(B) \frac{d B}{d t}
$$

where $B=\sqrt{B_{z}^{2}+B_{y}^{2}}$. The first term indicates saluration and the second hysteresis $|2|$. When the flux density is given, we calculate the waveform of the reluctivity during a single cycle and obtain in the Fourier expansion

$$
t(t)=\frac{H(B)}{B}=\nu_{0}+\sum_{n-1,2,3 \ldots} \nu_{n,} \sin (n \omega t)+\nu_{n e} \cos (n \omega t)
$$

where $\nu_{0}, \nu_{n c}$, and $\nu_{n c}$ are the coefficients of the fouricr transformation. We numerically conpute the coefficients when using the HBFEM.

The term $\partial \phi / \partial z$ of Eq.(2) is zero in the 2-dimensional case. However in order to satisfy the complementary condition, we assume that it is constant. Hence we have

$$
\iint_{S(\text { coil })} \sigma\left(-\frac{\partial A}{\partial t}-C\right) d x d y=0
$$

where

$$
C=\frac{\partial \phi}{\partial z}
$$

By substituting Eq.(4) into (7), we obtain

$$
C=\sum_{n-t, 2,3 \ldots} C_{n} i \sin (n \omega t)+C_{n c} i \cos (n \omega t)
$$

where

$$
\begin{aligned}
C_{n s} & =\sum_{\text {coil tement }} \frac{n \omega \Delta^{e}}{3 S}\left(A_{n c}^{1}+A_{n c}^{2}+A_{n c}^{3}\right) \\
C_{n c} & =-\sum_{\text {coil clement }} \frac{n \omega \Delta^{e}}{3 S}\left(A_{n d}^{1}+A_{n s}^{2}+A_{n s}^{3}\right) \\
S & =\sum_{\text {coil element }} \Delta^{*}
\end{aligned}
$$

$\Delta^{e}$ indicate the cross-section of an element e. By substituting Eqs.(4), (6) and (9) into (1), we carry out the formulation of HBFEM[1]. We have a matrix equation lor a single clement with the nodes $\left(x_{i}, y_{i}\right)(i=1,2,3)$

$$
\frac{1}{4 \Delta^{c}}\left[\begin{array}{lll}
\left(b_{1} b_{1}+c_{1} c_{1}\right) D & \left(b_{1} b_{2}+c_{1} c_{2}\right) D & \left(b_{1} b_{3}+c_{1} c_{3}\right) D \\
\left(b_{2} b_{1}+c_{2} c_{1}\right) D & \left(b_{2} b_{2}+c_{2} c_{2}\right) D & \left(b_{2} b_{3}+c_{2} c_{3}\right) D \\
\left(b_{3} b_{1}+c_{3} c_{1}\right) D & \left(b_{3} b_{2}+c_{3} c_{3}\right) D & \left(b_{3} b_{3}+c_{3} c_{3}\right) D
\end{array}\right]\{A\}
$$




$$
+\frac{\sigma w \Delta^{*}}{12}\left[\begin{array}{ccc}
2 N & N & N \\
N & 2 N & N \\
N & N & 2 N
\end{array}\right]\{A\}=\{K\}-\{R\}
$$

where

$$
b_{i}=y_{j}-y_{k}, c_{i}=x_{k}-x_{j}
$$

$\{A\}=\left\{A_{0}^{1} A_{1}^{1}, A_{1}^{1}, A_{2}^{1}, A_{2 \varepsilon}^{1} \cdots A_{0}^{2} A_{1}^{2}, A_{1,}^{2} A_{2,}^{2}, A_{2 c}^{2} \cdots\right.$ $\left.A_{0}^{3} A_{1}^{3}, A_{1}^{3}, A_{2}^{3}, A_{2}^{3}, \cdots\right\}^{T}$

$$
\begin{aligned}
& \{K\}=\frac{\Delta^{*}}{3}\left\{J_{0} J_{1}, J_{1 \varepsilon} J_{2}, J_{2 c} \cdots J_{0} J_{1,}, J_{1 \varepsilon}, J_{2}, J_{2 c} \cdots\right. \\
& \left.J_{0} J_{1}, J_{1 c} J_{2}, J_{2 c} \cdots\right\}^{T}
\end{aligned}
$$

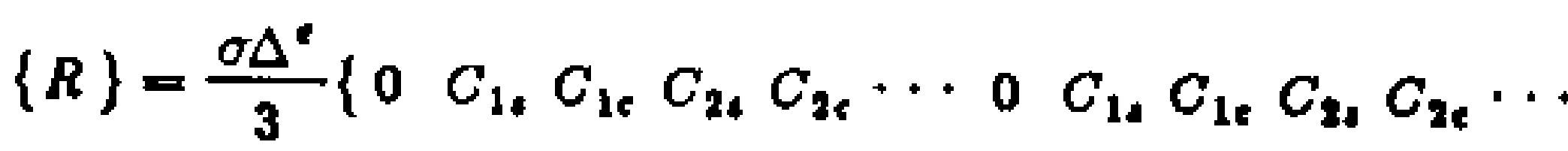

$$
\begin{aligned}
& \left.0 C_{1}, C_{16} C_{3}, C_{2 t} \cdots\right\}^{\mathrm{J}}
\end{aligned}
$$

The reluctivity matrix $D$ and the harmonic matrix $N$ are given by

$$
\begin{aligned}
& D=\frac{1}{2}\left[\begin{array}{cccccc}
2 \nu_{0} & \nu_{1}, & \nu_{1 c} & \nu_{21} & \nu_{2 c} & \cdots \\
2 \nu_{1}, & \nu_{0}-\nu_{2 c} & \nu_{21}, & \nu_{1 c}-\nu_{3 c} & -\nu_{1 c}+\nu_{3,} & \cdots \\
2 \nu_{1 c} & & 2 \nu_{0}+\nu_{2 c} & \nu_{1,}+\nu_{3,} & \nu_{1 c}+\nu_{3 c} & \cdots \\
2 \nu_{21} & & & 2 \nu_{0}-\nu_{4 c} & \nu_{4 t} & \cdots \\
2 \nu_{2 c} & & \text { symmetry } & & 2 \nu_{0}+\nu_{4 c} & \cdots \\
\vdots & \vdots & \vdots & \vdots & \vdots & \ddots
\end{array}\right] \\
& N=\left[\begin{array}{cccccc}
0 & 0 & 0 & 0 & 0 & \cdots \\
0 & 0 & 1 & 0 & 0 & \cdots \\
0 & -1 & 0 & 0 & 0 & \cdots \\
0 & 0 & 0 & 0 & 2 & \cdots \\
0 & 0 & 0 & -2 & 0 & \cdots \\
\vdots & \vdots & \vdots & \vdots & \vdots & \ddots
\end{array}\right]
\end{aligned}
$$

The structure of the system equation is identical to the one given in a previous paper[1]. Note that the reluctivity matrix $D$ and the harmonic matrix $N$ are changed.

We consider the formulation of the HBFEM in axisymmetric coordinates. Fortunately the process is the same as the one shown above. We have for a single element with the nodes $\left(r_{i}, z_{i}\right)(i=1,2,3)$

$$
\begin{aligned}
& \frac{r_{c}}{4 \Delta^{\prime}}\left[\begin{array}{lll}
\left(b_{1} b_{1}+c_{1} c_{1}\right) D & \left(b_{1} b_{2}+c_{1} c_{2}\right) D & \left(b_{1} b_{3}+c_{1} c_{3}\right) D \\
\left(b_{2} b_{1}+c_{2} c_{1}\right) D & \left(b_{2} b_{2}+c_{2} c_{2}\right) D & \left(b_{2} b_{3}+c_{2} c_{3}\right) D \\
\left(b_{3} b_{1}+c_{3} c_{1}\right) D & \left(b_{3} b_{2}+c_{3} c_{2}\right) D & \left(b_{3} b_{3}+c_{3} c_{3}\right) D
\end{array}\right]\left\{A_{1}\right\}
\end{aligned}
$$

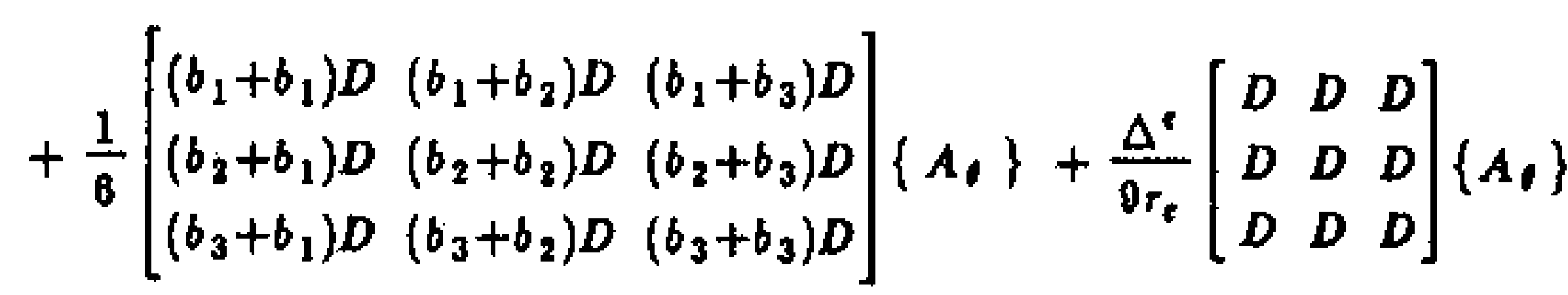

$$
\begin{aligned}
& +\frac{r_{e} \sigma w \Delta^{*}}{12}\left[\begin{array}{ccc}
2 N & N & N \\
N & 2 N & N \\
N & N & 2 N
\end{array}\right]\left\{A_{0}\right\}=\left\{K_{1}\right\}-\left\{R_{1}\right\}
\end{aligned}
$$

where

$$
b_{i}=z_{j}-z_{k}, c_{i}=r_{k}-r_{j}
$$

Here the vectors are given by

$$
\begin{aligned}
\left\{A_{0}\right\}= & \left\{A_{0}^{1} A_{1}^{1}, A_{1 \mathrm{c}}^{1} A_{2,}^{1} A_{2 c}^{1} \cdots A_{0}^{2} A_{1,}^{2}, A_{1 \mathrm{c}}^{2} A_{2,}^{2}, A_{2 c}^{2} \cdots\right. \\
& \left.A_{0}^{3} A_{1,}^{3} A_{1 \mathrm{j}}^{3} A_{2,}^{3}, A_{2 c}^{3} \cdots\right\}^{T} \\
\left\{K_{0}\right\}= & \left.\left.\left.\{\{K\}\}^{T}\{K\}\right\}^{T}\{K\}\right\}^{T}\right\}^{T} \\
\left\{R_{0}\right\}= & \left\{\left\{R^{\}}\right\}^{T}\left\{R^{3}\right\}^{T}\left\{R^{3}\right\}^{T}\right\}^{T}
\end{aligned}
$$

where

$$
\left\{K_{i}\right\}=\frac{\Delta^{e}}{4}\left(r_{\mathrm{c}}+\frac{r_{i}}{3}\right)\left\{J_{0} J_{1}, J_{1 c} J_{2,} J_{2 \varepsilon} \cdots\right\}^{T}
$$

$$
\begin{aligned}
& \left\{R,{ }^{i}\right\}=\frac{\sigma \Delta^{e}}{3}\left(0 C_{1}^{i}, C_{1,}^{i} C_{2,}^{i}, C_{2 e}^{i} \cdots\right\}^{T} \\
& C_{n s}^{i}=\sum_{\text {coil eliement }} \frac{n \omega \Delta^{*}}{4 S} \sum_{i=1,2,3}\left(r_{c}+\frac{r_{i}}{3}\right) A_{n c}^{i} \\
& C_{n c}^{i}=-\sum_{\text {coil setement }} \frac{n \omega \Delta^{e}}{4 S} \sum_{i=1,2,3}\left(r_{c}+\frac{r_{i}}{3}\right) A_{n s}^{i}
\end{aligned}
$$

The block matrices $D$ and $N$ are idenlical to the ones in Eqs.(13) and (14).

\section{VERIFICATION OF THE FORMULATION}

Let us consider the eddy current-free smoothing reactor with an air gap as shown in Fig.1(a). The magnetic core is composed of $\mathrm{Mn}-\mathrm{Zn}$ ferrite with the magnetizing curve shown in Fig.1(b). The hysteresis is not taken into consideration in this model. Figs.2 and 3 show the flux distribution for each harmonic and the waveform of flux density when the waveform of the magmelizing current is approximately triangular as shown in Fig. 3. 'T'he calculation takes into account - all harmonics up to the 5 - th.

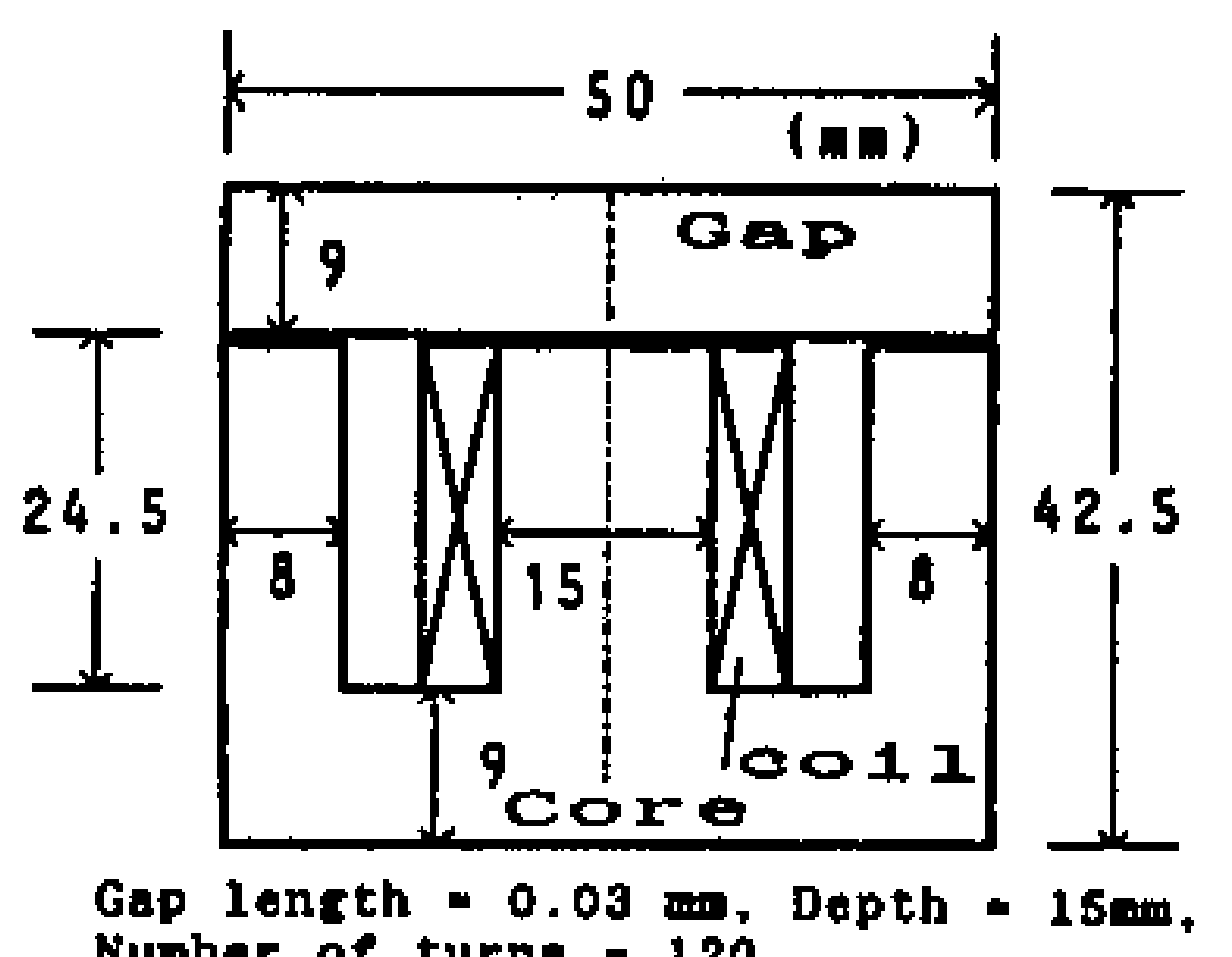

Gap 1ength -0.03 mas, Depth $-15 \mathrm{~mm}$

(a) Confguration and dimensions

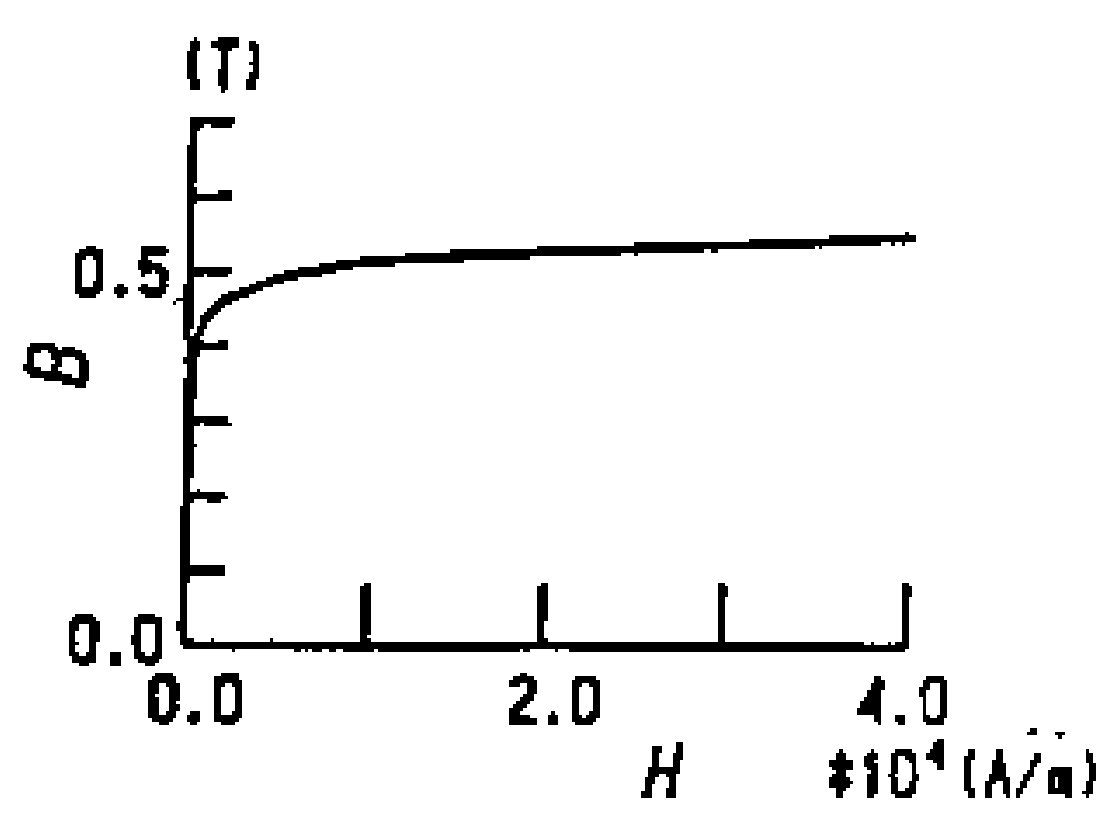
(Mn-Zn ferrite) (b) Magnetizing curve

Fig.1 Smoothing reactor

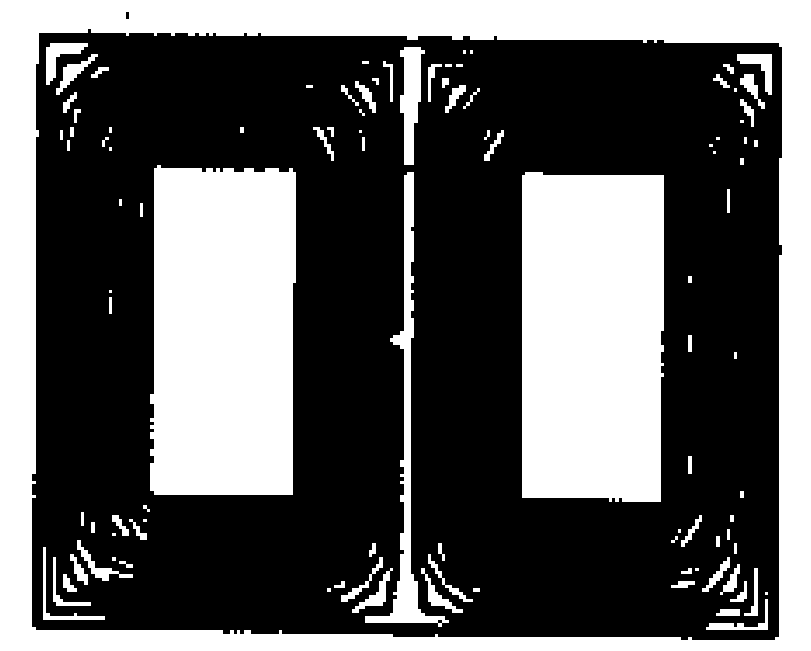

Dc-component

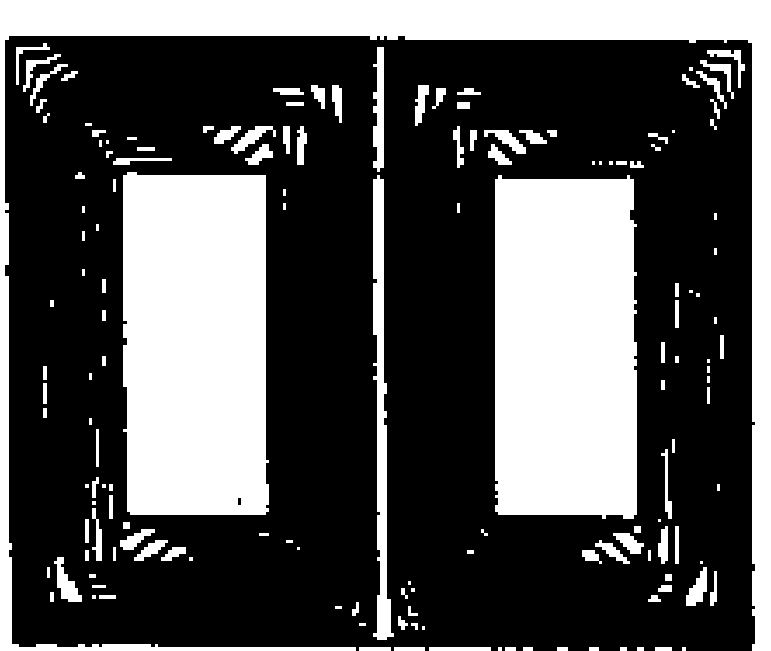

fundamental

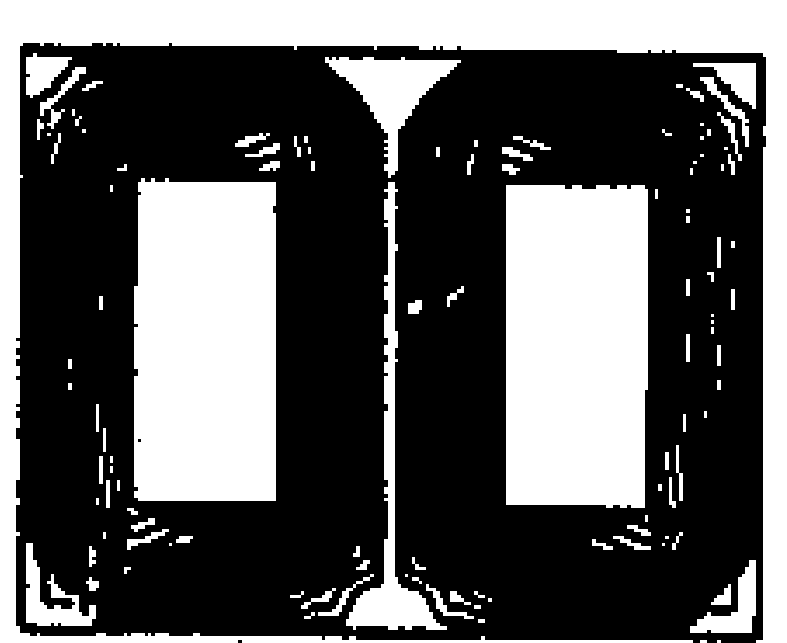

2-nd harmonic

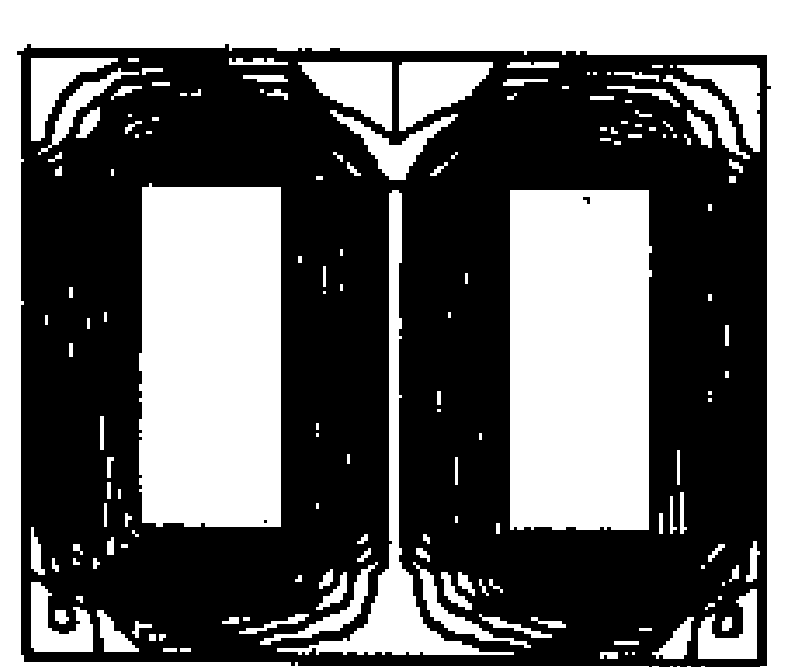

3-rd harmonic

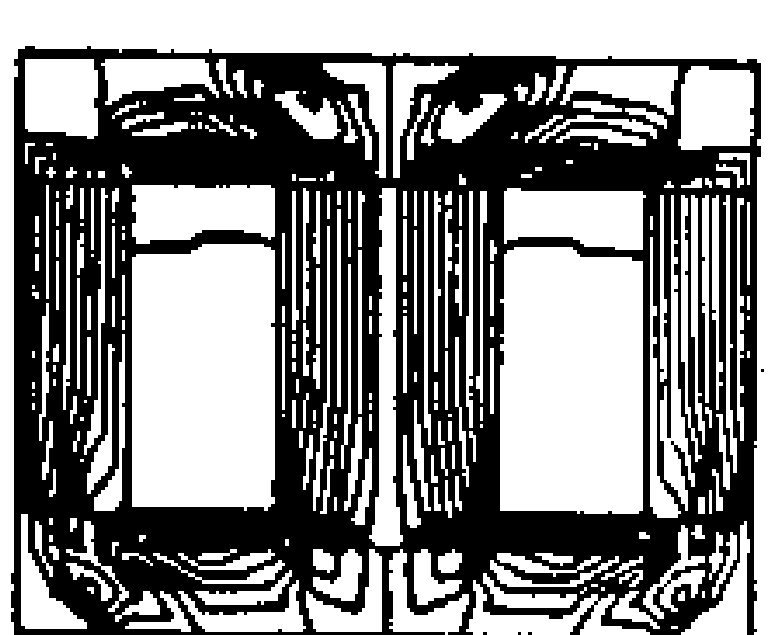

4-th harmonic

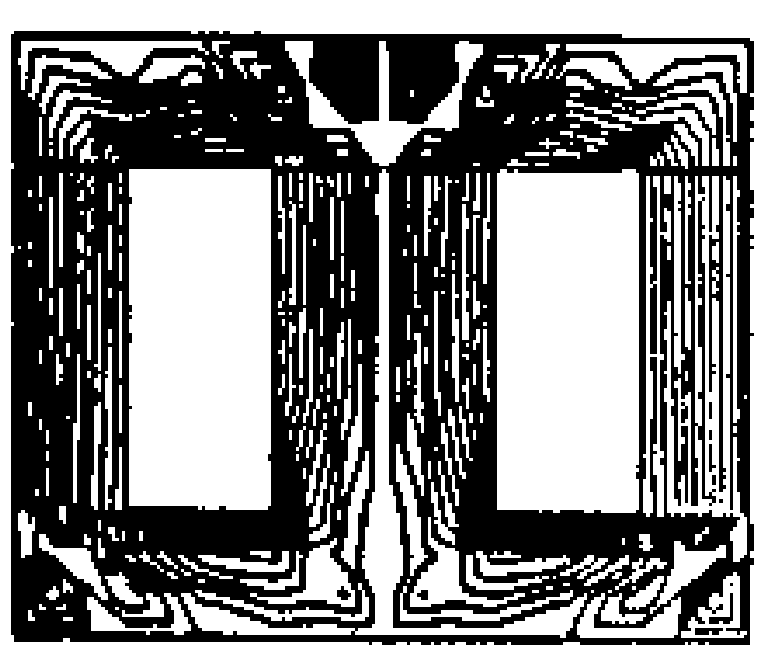

5-th harmonic
Fig.2 Flux distribution of each component of magnetization

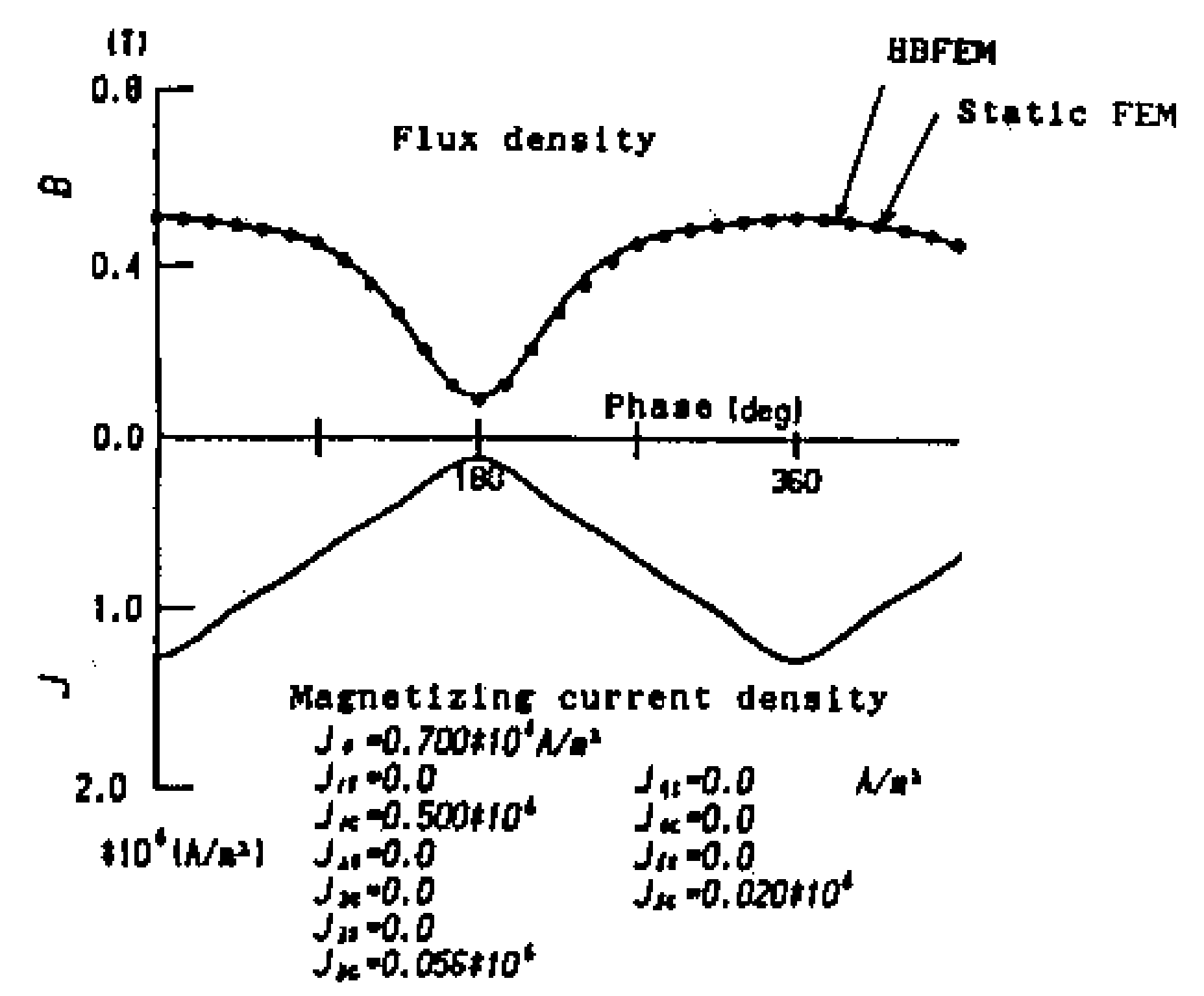

Fig.3 Waveform of flux density in the core 

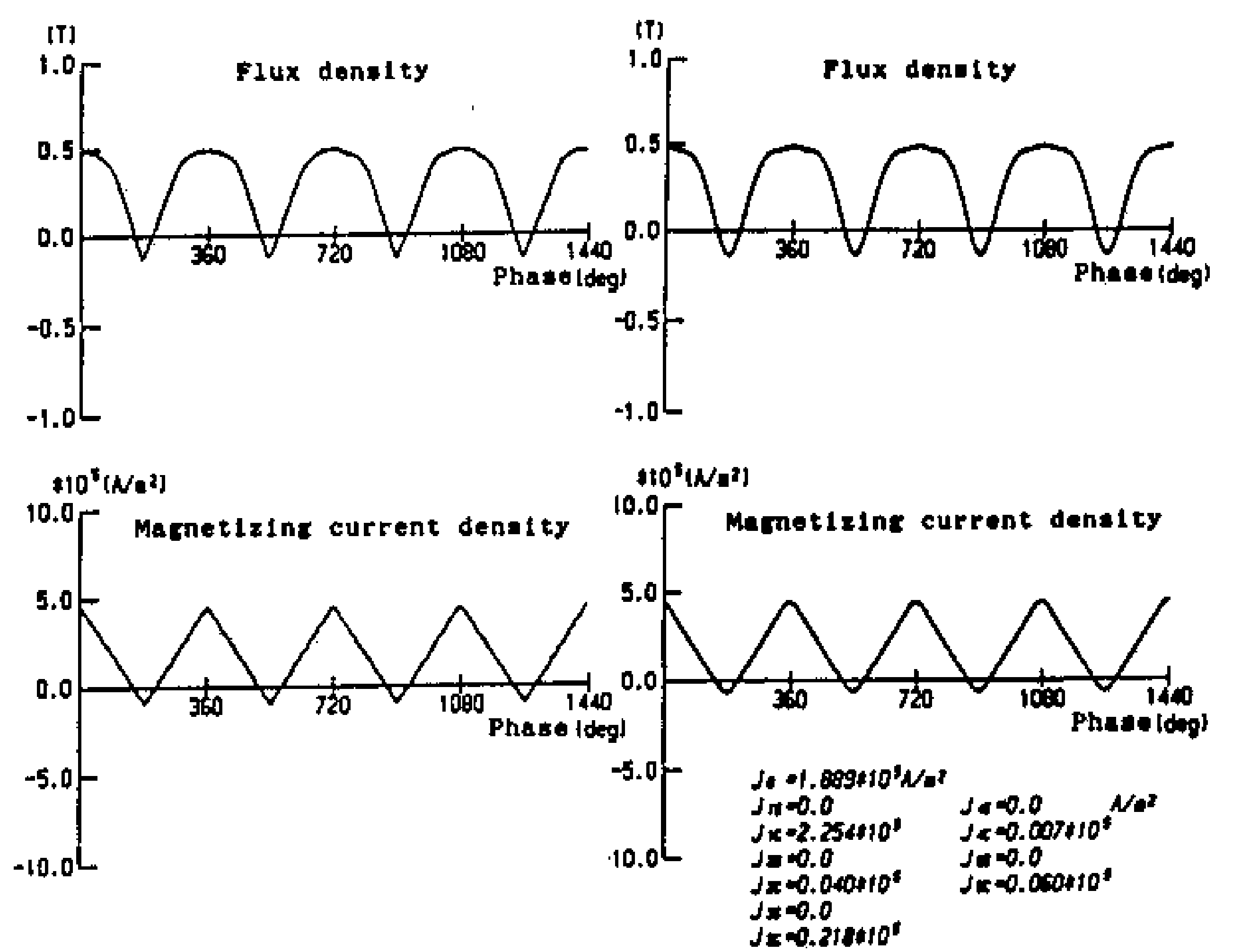

(a) Experimental results

(b) Calculated results

Fig.4 Comparisons of flux density waveforms

The circles in Fig. 3 indicate the calculated values obtained by the static FEM[3]. Good agreement exists between the values obtained by the FEM and the HBFEM.

Fig.4 shows the comparison between the calculated and the experimental results. The harmonics of current density up to the 5-th are considered in the HIBIEM. The dc magnetization yields an asymmetric waveform of flux density in spite of the triangular magnetizing current density waveform.

\section{APPLICATION TO HIGH FREQUENCY TRANSFORMERS}

Fig.5 shows the axisymmetrical pot-core transformer of a high frequency resonant-type converter. The transformer has relar tively high wide air-gap to store the resonant energy. Due to leakage flux, eddy currents are induced in the low-voltage winding. As the cross-section of the high-voltage primary winding is sinaller than that of the secondary, it is sufflcient to consider the eddy currents in the secondary winding. The operating frequency is about $1 \mathrm{MHz}$. Fig.6(a) shows the B-H curve at the operating frequency. Based on the measured data, we approximate the magnetizing curve with hysteresis by Eq.(19):

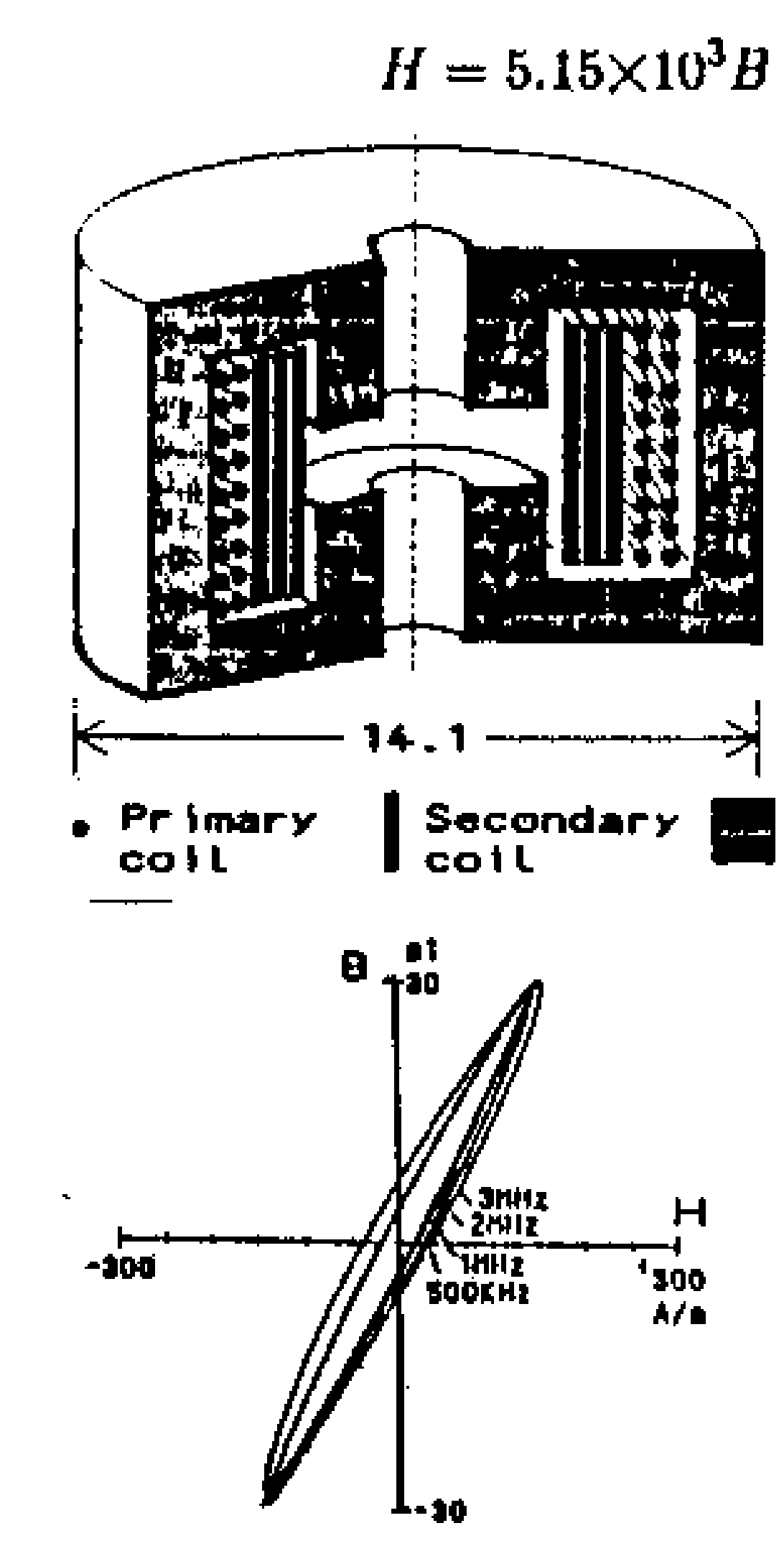

(a) Measured curve

\footnotetext{
Fig.6 Magnetizing curve
}

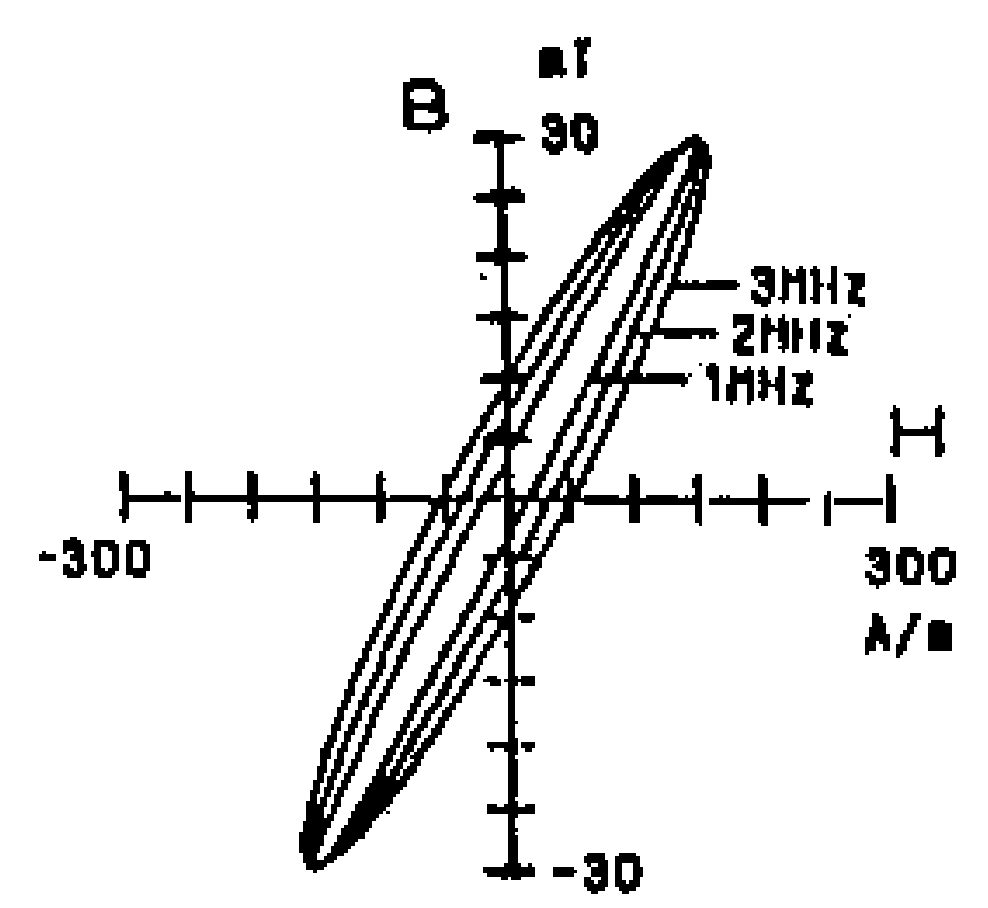

(b) Approximated curve
Fig. 5 Pot-core type transformer design.
The approximated B-H curve is shown in Fig.6(b).

Fig.7 shows the flux distribution of each harmonic. In this analysis, both de and ac components up to the 2-nd harmonic are taken into consideration. Note that the eddy currents in the low-voitage coil prevent the fundamental flux leakage al the air-gap. Fig.8 shows the current density distribution in the coil. The dotted line indicates the average current density. Since the total current equals to the applied current, the result shows high current density at the surfaces of the coil. Unfortu nately, this distribution yields high eddy-current losses.

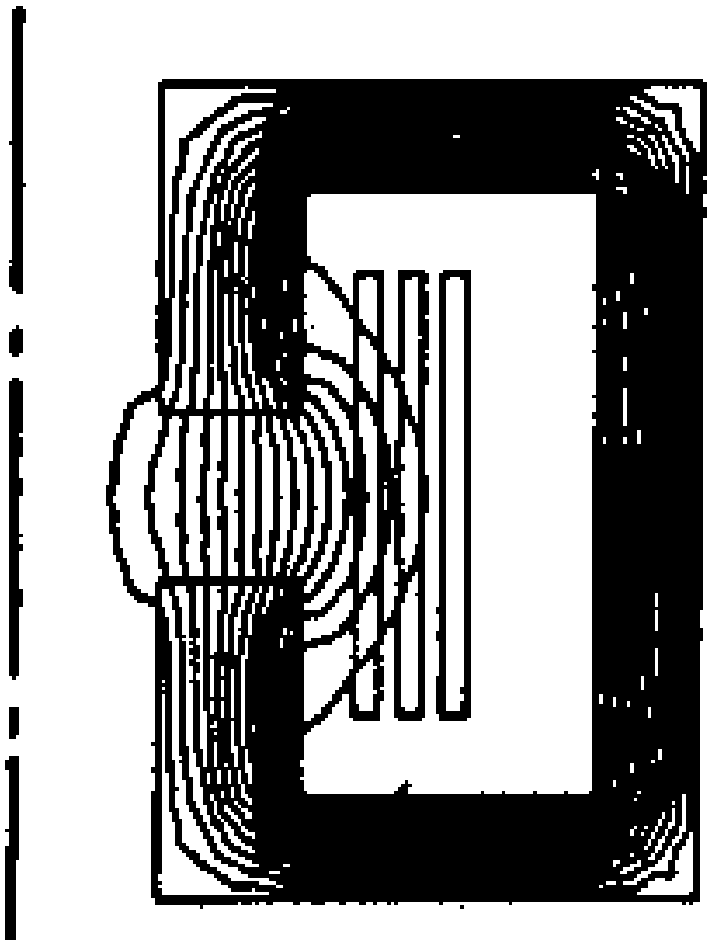

(a) Dc component

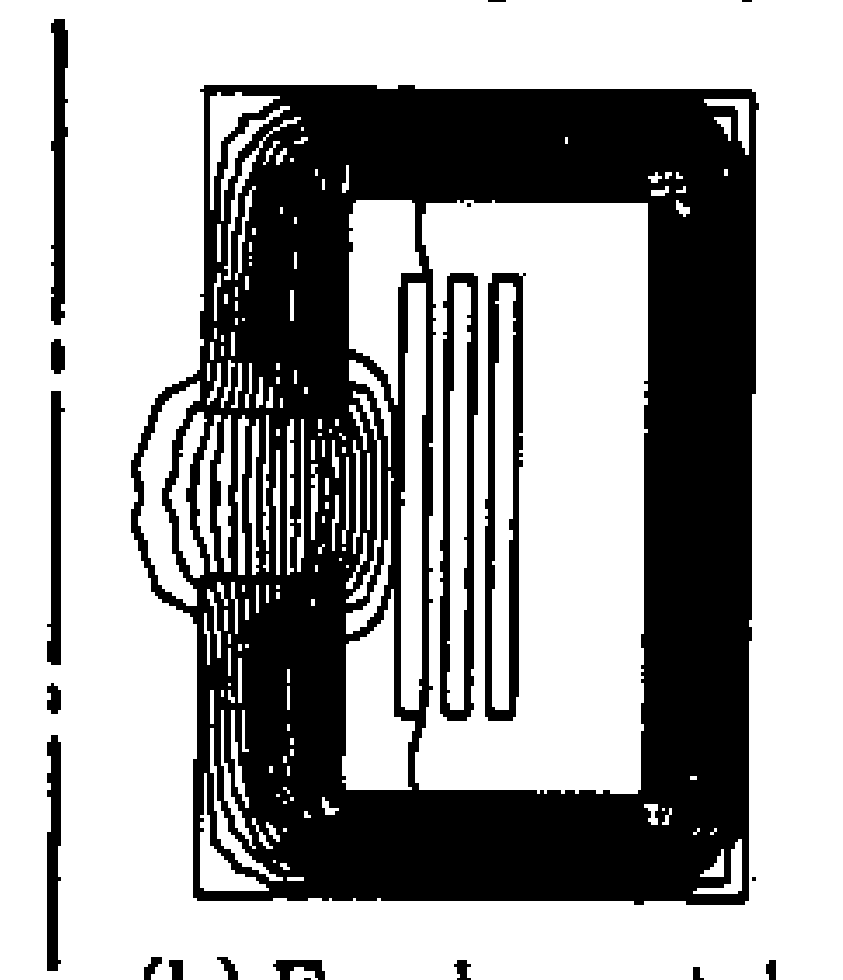

(b) Fundamental

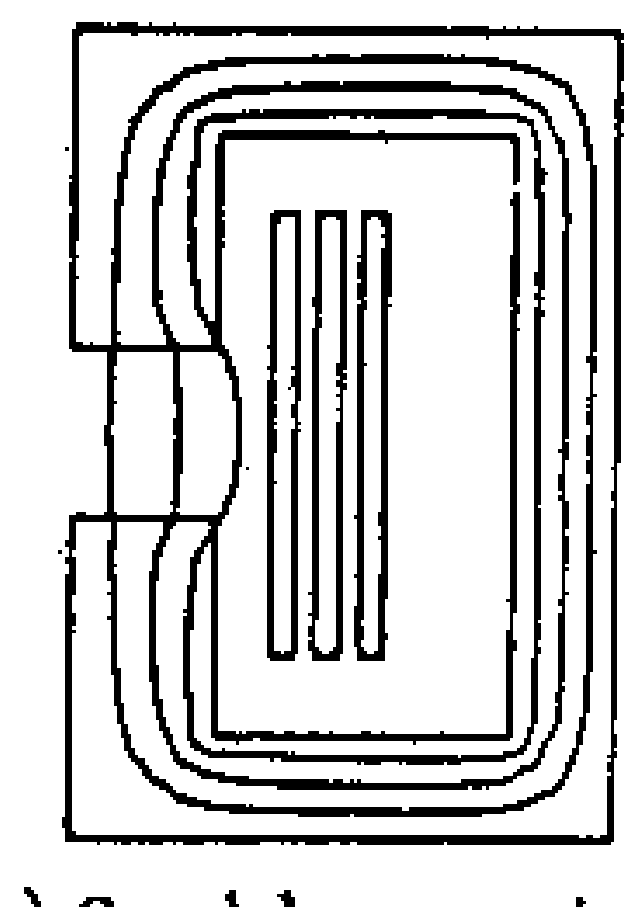

(c) 2-nd harmonic
Fig.7 Flux distributions

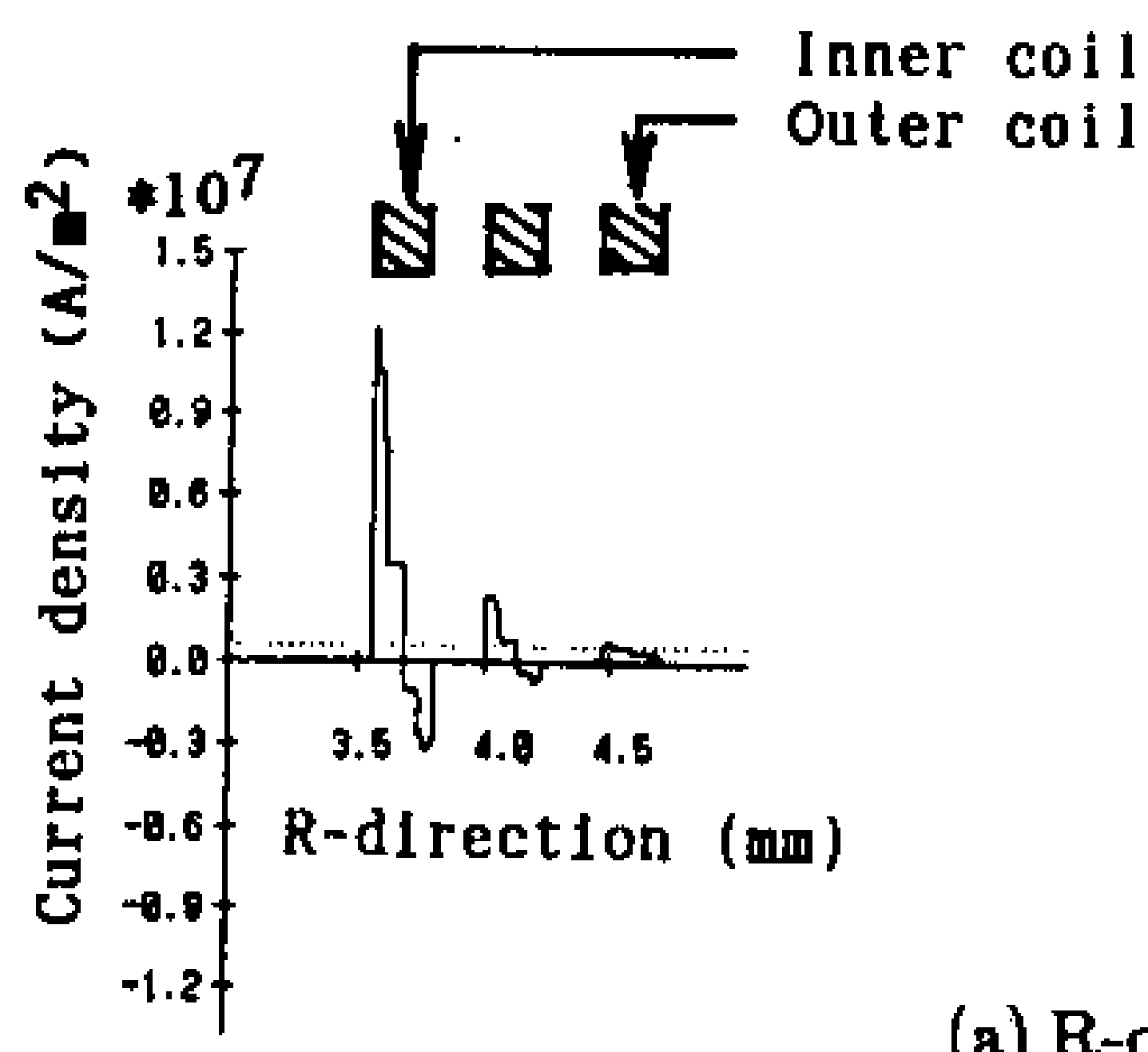

(a) R-direction distribution

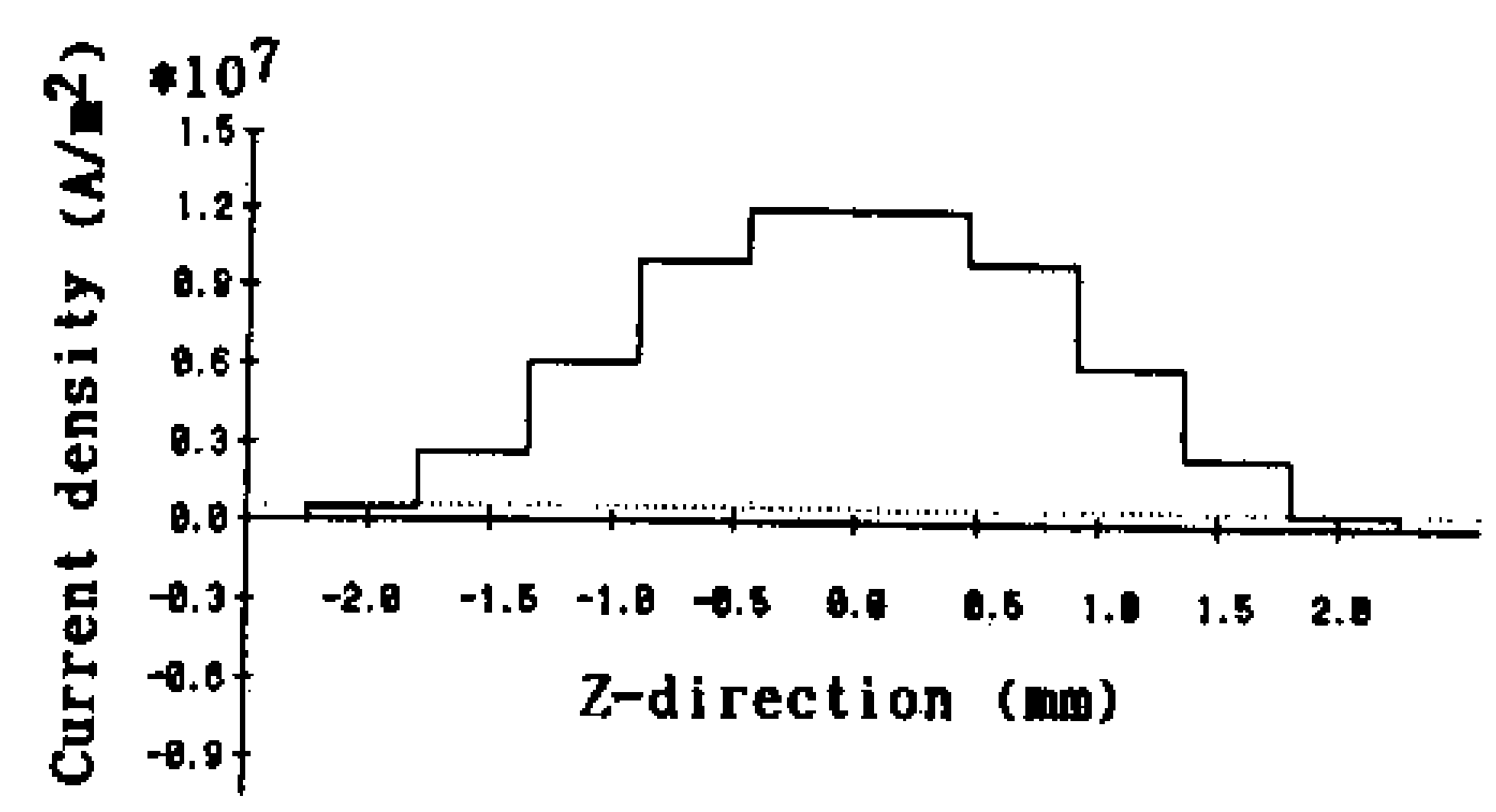

(b) Z-direction distribution in the surface of the inner coil

Fig.8 Distribution of current density

\section{CONCLUSIONS}

We proposed a new type of finile elernent analysis in the harmonic domain. The merit of the HEFEM is that the calculated results of the frequency characteristics are applicable for

\section{REFERENCES}

[1] S.Yamada, K.Bessho, J.Lu, "Harmonic Balance Finite Element Method Applied to Nonlinear AC Magnetic Analysis", IEEE Trans. Magnetics, Vol.MAG-25, No.4, pp95-99(1989).

[2] Y.Saito, S.Hayano, T.Yamamurs, N.Truya, "A Representation of Magnetic Hysteresis", IEEE Trans. Magnetics, Vol.MAG-20, No.5, ppl434. 1438(1984).

[3] P.Silvester, M.V.K.Chari, "Finite Element Solution of Saturable Magnetic Field Problems", IEEE Trans. PAS, Vol.PAS-89, No.7, pp 1642 . 1649(1970). 